\title{
Interval Subroutine Library Mission ${ }^{\star}$
}

\author{
George F. Corliss ${ }^{1}$, R. Baker Kearfott ${ }^{2}$, Ned Nedialkov ${ }^{3}$, John D. Pryce ${ }^{4}$, and \\ Spencer Smith ${ }^{5}$ \\ 1 Marquette University \\ 2 University of Louisiana at Lafayette \\ 3 McMaster University and Lawrence Livermore National Laboratory \\ ${ }^{4}$ Cranfield University, RMCS Shrivenham \\ ${ }^{5}$ McMaster University
}

\begin{abstract}
We propose the collection, standardization, and distribution of a full-featured, production quality library for reliable scientific computing with routines using interval techniques for use by the wide community of applications developers.
\end{abstract}

\section{Vision - Why are we doing this?}

The interval/reliable computing research community has long worked to attract practicing scientists and engineers to use its results. We use any of the terms interval, reliable, verified computation in the sense of producing rigorous bounds on true results, e.g., [1,2]. The Interval Subroutine Library (ISL) is a project to place interval tools into the hands of people we believe will benefit from their use by gathering and refining existing tools from many interval authors. We acknowledge that intervals carry a steep learning curve, and that they sometimes have been over-promised. The winning strategy for widespread adoption of interval technologies is the development of "killer applications" that are so much better (in some sense) than current practice that practicing scientists and engineers have no choice but to adopt the new technology.

The ISL team wants to see such killer applications appear, but producing them is not our mission. The routine use of interval techniques by practicing scientists and engineers is hampered by a lack of widely-used, comprehensive, quality interval software that is available on all major platforms (Linux, Mac, Unix, Windows). Once such software is available, use of interval techniques is likely to grow along at least three paths: small-scale applications by scientists/engineers in the course of their daily work; professionally built applications in a specific area, such as global optimization or curve graphing; and the almost invisible embedding of verified computing as a tool in commodity software such as spreadsheets or scientific data analysis and document preparation.

\footnotetext{
* This work was supported in part by EPSRC Grant D033373/1. Submitted to Reliable Implementation of Real Number Algorithms: Theory and Practice, in the LNCS series of Springer Verlag, P. Hertling, C. Hoffmann, W. Luther, and N. Revol, eds.
} 
ISL can provide the infrastructure for such developments. ISL targets application developers, those who are developing the significant applications. Intervalbased tools tailored for specific end-practitioner applications are developed by applications developers with expertise in applications areas, but those developers are not finding interval tools they perceive as attractive for their applications. Currently, if we talk to a group of scientists or engineers about intervals and convince them of the value of interval techniques, when they ask, "Great! What software can I use?" there is a long, painful pause. We have many tools, packages, and research codes, but we have no CD that solves their problems with rigorous bounds.

The ISL project itself does not author software; contributing authors do that. The goal of the ISL project is to gather and disseminate a library of high quality interval-based tools. The fundamental requirements is, "Thou shalt not lie." Routines are expected to return an enclosure of the correct mathematical result or else provide a suitable indication of failure. The qualities of interest for the ISL project include

$$
\begin{array}{ll}
\text { - } \operatorname{correctness,} & \text { - comprehensiveness, } \\
\text { - } \text { reliability, } & \text { - performance, } \\
\text { - } \text { robustness, } & \text { - maintainability, and } \\
\text { - } \text { usability, } & \text { - portability. }
\end{array}
$$

To achieve these qualities, the ISL project encourages its contributing authors to use software engineering sound principles, including documentation, good architecture, thorough testing, and coding standards. The documentation produced and the process of assembling the library also support the goal of achieving high quality. Documentation should be complete, consistent, correct, usable, verifiable, maintainable, and reusable. The development process should have the qualities of productivity, timeliness, and transparency.

The authors are embarking upon a plan for the cooperative development of such a library. This paper lays out the broad scope of the project.

\subsection{Short-term goals}

By the end of 2007, we expect to offer interval BLAS levels 1 and 2 and a collection of problem-solving packages, mostly chosen from existing software, including linear systems, optimization, and differential equations. The collection may include utilities for automatic differentiation, Taylor models, and constraint propagation. For our plan to achieve this, see $\S 3.1$.

\section{$1.2 \quad$ Long-term goals}

In perhaps 6 to 8 years, we hope to offer a library of interval tools with coverage comparable to early releases of the IMSL or NAG libraries, SLATEC [3], the popular Numerical Recipes books [4,5], the GSL - GNU Scientific Library [6], or other comparable libraries. The library will be freely available, and we shall also 
encourage its appearance in commercial products. The library should be used by a significant number of applications widely used in their respective domains. For our plan to achieve this, see $\S 3.2$

\section{Product - What will we deliver?}

To meet the needs of a wide community of applications developers in a broad cross-section of applications areas, we need a portable, comprehensive, production-quality library of interval tools solving most of the standard problems of scientific computation. The library should be available in a downloadable or CD form. The library should also have a clear licensing structure that protects authors, while still encouraging commercialization.

\subsection{Contents}

We envision a hierarchical library, with units organized into chapters roughly as suggested by Figure 1:

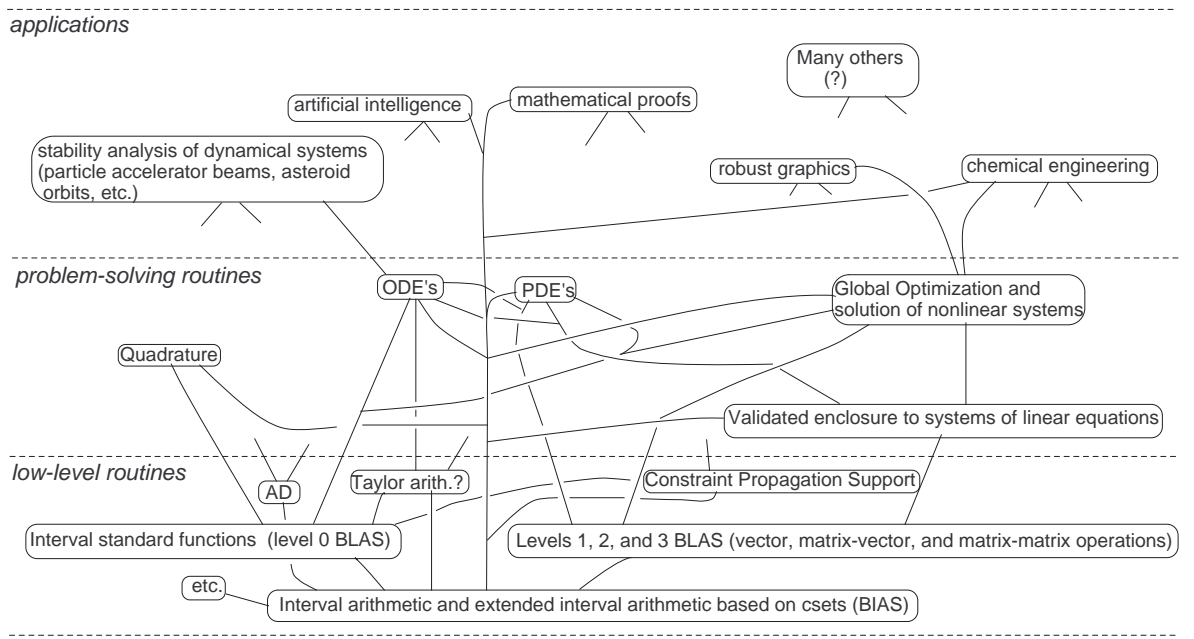

Fig. 1. A tentative hierarchical structure.

Level 0 - Basic Interval Arithmetic: Interval arithmetic including

1. Constructors,

2. Arithmetic operations,

3. Comparison operators,

4. Input/output, and

5. Elementary functions. 
Level 0 should be consistent with the $\mathrm{C}++$ interval arithmetic standard [7] proposed by Brönnimann, Melquiond, and Pion (BMP) and have a large overlap with the functionality provided by many current interval arithmetic packages, including PROFIL/BIAS [8], FILIB++ [9], Boost [10], Gaol [11], C-XSC [12-16], and the Sun C++ compiler [17].

Level 1 - Utilities: Level 1 units are called by several units in Level 2 to provide capabilities including

1. Error handler,

2. Additional (non-basic) interval arithmetic features,

3. Vectors and matrix classes,

4. Level 1 and level 2 BLAS,

5. Automatic differentiation,

6. Taylor model arithmetic, and

7. Constraint propagation.

Level 2 - Problem-solving routines: Chapter outline will grow with time, initially, similar to the contents of many textbooks in numerical analysis:

1. Linear systems, eventually including sparse and eigensystems,

2. Nonlinear systems,

3. Optimization,

4. Quadrature,

5. Statistics,

6. Ordinary differential equations, and

7. Partial differential equations.

Level 3 - Applications: Not in the scope of ISL, but we strongly encourage applications developers to build on ISL.

Capabilities not listed here are by no means left out. For example, Level 1 may include multiple precision interval arithmetic with an API close to the Level 0 , so that Level 2 and Level 3 units can easily switch from double precision intervals to intervals of higher precision, or vector and matrix classes using elliptical representations for multi-dimensional intervals. Eventually, each chapter should contain a variety of general- and special-purpose routines. Categorizing interval software in a structure roughly paralleling widely-used approximate libraries encourages interval researchers to consider gaps in interval coverage.

\section{Plan - How will we accomplish that?}

We have both short-term (two years) and long-term (3 - 10 years) plans.

\subsection{Short-term plan: gather, organize, and disseminate}

For perhaps two years, this is primarily a library project in the sense of identifying, collecting, organizing, and making available work that already exists: 
Step 1 - Language standardization. Brönnimann, et al. have proposed to add intervals to the $\mathrm{C}++$ language standard [7]. The ISL team is working for the strengthening and the adoption of this proposal. The BMP proposal can become the basis for our ISL Level 0 BIAS well before it is approved. Several existing implementations of intervals in $\mathrm{C}++$ are reasonably close to the proposed standard, so multiple (almost) reference implementations are available.

Step 2 - Pilot inclusion into ISL. Select about three existing packages for initial inclusion into ISL. This gives us a chance to prototype policies, procedures, and practices for incorporating existing work. See the discussion of some issues in $\S \S 3.3-3.9$.

Step 3 - Invite participation. Once some of the issues of policies, procedures, and practices for incorporating existing work have been refined, we will invite 6-10 researchers to submit their work for inclusion in ISL. At this stage, the number of packages we will invite remains modest, as we develop experience, participation, and visibility.

We hope for a very preliminary release including parts of Steps 1 and 2 by the end of 2006 and a release including about five ISL Level 2 problem-solving routines by the end of 2007 .

\subsection{Long-term plan}

After we gain experience and visibility from the short-term "gather, organize, and make available" activities, we expect to expand the scope of the library by inviting contributions from the interval community. Work will be managed along the model of many successful open source projects. We anticipate releases each 1-2 years. We will continue development of a free version of ISL, while seeking a commercial partner such as NAG, Sun, IBM, Intel, or Microsoft.

Next, we turn our attention in subsections 3.3-3.9 to some of the issues that must be settled to ensure the success of ISL.

\subsection{Language and environment}

We do not wish to ignite religious warfare, but we must choose an appropriate computer language for ISL. In the short-term "gather, organize, and make available" activities, we can include packages in any language. Most existing interval software is in Matlab or some dialect of Fortran or C++. ISL is in C++ because there appears to be more existing interval software in $\mathrm{C}++$ than in other candidates.

In the future, we may employ generative programming techniques, similar to the NAG engine. The code could be written in one language and automatically "compiled" into whatever source code languages are supported. The NAG specification language is very similar to Fortran. 


\subsection{Organizational structure}

Quality, comprehensive libraries are not compiled by a single person or small group of people over a short time. There are many models we can follow of software development by large, loosely-coupled teams over several years, including the LAPACK project [18], PETSc [19-21], and many open source projects such as the GSL - GNU Scientific Library [6].

The ISL project is coordinated by a steering committee, currently, the authors of this paper. We meet occasionally as a group, and subsets meet as possible at conferences. The steering committee sets directions and policies, such as those outlined in this paper. In the long-term steady state, the role of the steering committee is somewhat like that of the editorial board of a major journal, overseeing the work of authors, referees, and the publication process.

\subsection{Adding value}

There are several good packages for interval arithmetic corresponding to our proposed Level 0 BIAS, there are many interval-based problem-solving routines corresponding to our Levels 1 and 2, and there are a few comprehensive projects such as Karlsruhe XSC Toolbox books [12] and Neumaier's COCONUT (http: //www.mat.univie.ac.at/ neum/glopt/coconut/). Vladik Kreinovich does an admirable job of capturing and maintaining pointers to many interval projects at [22]. What value does ISL add?

We return to our initial premise. Although there many interval tools are available, there is no single source, a web site or a CD offering a standardized, portable, peer-reviewed suite of tools that install and work together. In the long term, we envision a comprehensive, universally used library. This is in contrast to offering general languages, such as in the COCONUT project or GAMS, or offering graphical user interfaces, such as in commercial packages such as Maple. We view the effort as promoting standardization, portability, and re-use. In the short term, ISL works with contributing authors to gather existing interval tools, standardize their installation and interfaces, perform peer review acceptance and comparative testing, provide examples, and make these tools available from a single source.

\subsection{Quality Assurance}

All interval code has to have the quality of correctness. The code must obey the rule "Thou shalt not lie."

Contributions are peer refereed. To be considered for inclusion in ISL, normally we expect the algorithm to have been the subject of at least one peer refereed journal paper. Codes, testing, and documentation are also refereed, similar to the standards for an ACM Algorithm. We intend that publications and programs associated with ISL be held to the highest academic and software engineering standards. 
We strongly recommend that contributing authors follow modern software engineering practices, recognizing that "modern software engineering practices" encompass methods proposed by Parnas [23], Literate Programming [24,25], agile methods such as Extreme Programming [26] and Test-Driven Development [27], and others. Generally, we favor the more formal methods because specifications for, say, a linear equation solver, are not expected to change significantly while development is being done.

The ideal contribution to ISL consists of the following:

1. A specification of the software requirements, including the mathematical statement of the problem and information on the required inputs and possible output values. With respect to the inputs, the specification indicates clearly any constraints that exist on the data. Where necessary, a flag shall be specified whose values indicate the reason for failure when a solution cannot be determined. All contributions to ISL share the goal of achieving the qualities listed in the introduction, especially the requirement, "Thou shalt not lie." However, it is difficult to write validatable specifications of nonfunctional requirements. For instance, validating correctness is challenging for scientific computing problems, because formal proofs of correctness are difficult and often overly conservative, although non-formal proofs with rigor comparable to proofs in the mathematical literature are often appropriate. Therefore, rather than specify the requirements, the approach is taken of describing the final software product, typically including statements of the form, "It finds an enclosure of correct solution if the input lies in set $Y$." This description is given in the software validation report, discussed below.

2. A design specification. The ideal specifications includes an API or function signature plus semantics, often modeled on specifications for corresponding packages for approximate solutions.

3. A software validation report. The software validation report is about a combination of observed scope, tightness and speed (plus maybe memory load), and the observed interplay among them. It characterizes the problems that are successfully solved.

The contributing authors are asked to provide the evidence that the software meets the stated requirements and to describe the level to which the software meets the software quality goals. The software validation can consist of informal and formal analysis, testing and a summary of important software metrics. Techniques for informal and formal analysis include code walkthroughs, code reviews, and inspection. Techniques such as literate programming can be employed so that confidence can be built on the correctness of the code, in a similar sense to how confidence is built by mathematicians inspecting a mathematical proof. The summary of testing also builds confidence by showing the test cases that were passed and that any user can download and run for themselves. The descriptions set expectations for the behavior of the program in similar situations. The descriptions can be tested for lies; for instance, the validation report might assert, "the software was run over a given range of inputs on machines $x$ and $y$ and the program ter- 
minated in 5 seconds or less with an enclosure of the correct answer in $87 \%$ of cases, terminated in 5 seconds or less with a failure indicator in $10 \%$ of cases, and had not terminated in 5 seconds in the remaining $3 \%$ of cases."

\subsection{Licensing}

Especially in view of Sun being granted several interval-related patent applications, the interval community is increasingly aware of the importance of the protection of intellectual property. ISL needs a carefully considered license which

- protects rights and reputations of authors,

- provides for free distribution, and

- encourages commercialization.

To help us in that, we are gathering and evaluating examples including

- LGPL, Modified Berkeley, MIT, and other Open Source licenses;

- licenses of various interval packages; and

- intellectual property policies of some (possibly) participating universities.

\subsection{Publications}

Since many potential participants in the ISL project are academics, the project will not succeed without clear publication opportunities:

- Continuing publication of incremental and innovative development of interval software;

- Identification of omissions in coverage (holes) as development targets;

- Comparative testing of similar packages in the spirit of Enright and Hull [28] or Mazzia, Iavernaro, and Magherini [29] for approximate ODE solvers or Pryce [30,31] for Sturm-Liouville solvers; and

- Suites of test problems for interval problem-solving routines, e.g., Corliss and $\mathrm{Yu}$ for interval arithmetic operations and elementary functions [32]. Interval test suites should include many problems from existing test suites for approximate solvers and also problems intended to test existence and containment properties.

- Software engineering publications related to the development of scientific software with respect to appropriate process models, methodologies and documentation. Software engineering has mostly ignored scientific software and placed most of the emphasis on research on safety critical systems and information systems. There is room to make contributions by looking at the issues that are specific to scientific software.

For academic researchers, release of software to ISL in addition to journal publications offers an external, peer-reviewed process for recognizing research contributions and wider dissemination than links from the authors' web site.

While valuing the role of Reliable Computing as the core journal in this research field, we encourage contributing authors to publish in a wide variety 
of journals, especially journals in applications areas, to help bring the message of intervals to as wide an audience as possible. Intervals are much closer to the main stream of scientific computing than many of us realize, as new applications and researchers using interval techniques are published regularly. We help more people learn about intervals by publishing in the outlets they read.

In the long-term steady state, having code accepted for inclusion in ISL may be viewed as equivalent to a journal paper, probably contributing more to the overall advance of the infrastructure of science than many journal papers.

\subsection{Funding}

While it would be welcome if someone wanted to provide large funding, that is unlikely. If we look at the models of LAPACK or most open source projects, there may be modest funding somewhere for overall leadership and organization, but the developers are on their own to secure their own funding. One would hope that contributing to a large, well-organized, well-publicized international effort might help many interval researchers get our own work funded.

Similarly, it would be welcome if a large software company provided the leadership and modest funding for the champion to lead an open source project. In other fields of study, with more obvious customers, several firms have made significant contributions to various open source projects.

\section{Partners - How can you help?}

Clearly, the long-term goal is ambitious, requiring the work of many people over many years. This section outlines our vision of an ideal partnership of contributing authors, chapter architects, referees, and ISL steering committee.

\subsection{Contributing author}

A contributing author contributes any of

- Code unit for the library to solve some well-defined problem of scientific computing, e.g., constraint propagation, linear systems, optimization;

- Functionality or performance improvements, corrections, or extensions to an existing unit of the library;

- Test suites;

- Documentation; and

- API architecture for a chapter of the library.

For a new unit for the library, a contributing author should submit

- User Guide: installation, requirements, examples;

- Maintenance documentation: system architecture, detailed design, test plan and report;

- Source code; and 
- Journal article (with quality of TOMS article and algorithm).

A typical interaction might be

1. Contributing author contacts (or is contacted by) the ISL steering committee.

2. They discuss a suitable problem of scientific computing, specifications, licensing, etc.

3. Contributing author submits a suitable research publication to a journal.

4. Contributing author submits to ISL source; installation instructions; documentation of the problem, description of algorithms, examples of use, references, etc.; acceptance and other tests; copies of journal papers, etc.

5. ISL or chapter editor sends submitted materials to referees.

6. Usual iterations with editors, referees, and authors.

7. ISL accepts or declines the submission.

8. After acceptance, ISL maintains discussions with the contributing author to ensure that updates to the original work are reflected in the library.

ISL should be more than a listing of web links to contributing authors' pages. That requires some process, at least semi-formal. The short-term "gather, organize, and make available" phase of the project will be used to find an appropriate balance of a formal process to ensure quality and a light-weight process all can use effectively. For example, there is no need to duplicate refereeing work already performed for journal publication.

\subsection{Chapter architect/editor}

In the short term, the ISL steering committee are the architects of the library and the editors for contributed units. As the scope grows, each chapter of the library (see $\S 2.1$ ) has an architect/editor responsible for

- External architecture of the chapter, problem coverage, consistent API;

- Internal architecture, shared utilities; and

- Collaboration with contributing authors for this chapter.

\subsection{Referee}

The referee contributes to the overall quality of the library by providing an external assessment. The referee reviews materials submitted by contributing authors including source, installation instructions, documentation of the problem, description of algorithms, examples of use, references, acceptance and other tests. The referee is assessing the library materials as they affect application developers who use the library, rather than the more academic concerns of a traditional journal referee. We anticipate that some referees are anonymous, and some are collegial.

The ISL refereeing process may be modeled on the process for refereeing ACM Algorithms. We encourage ISL contributing authors to submit their work as ACM Algorithms, in which case, the ISL refereeing is sharply truncated. 
We anticipate that some refereeing work leads to publishable careful comparative testing of similar packages and compilation of sets of standard test problems for interval problem-solving routines.

\subsection{Applications development}

The goal of the ISL project is to get quality, portable, uniform, comprehensive interval tools into use by developers of applications used by practicing scientists and engineers. Our target audience includes

- Developers in the interval community, our contributing authors. For example, authors of interval DE or optimization solvers benefit from AD, constraint propagation, and linear solvers in ISL.

- Scientists from applications areas developing more reliable software than that currently available, e.g., Martin Berz and Kyoko Makino (theoretical physics), Mark Stadtherr and Paul Barton (chemical engineering), Rafi Muhanna and Robert Mullins (structural engineering), and William Edmunson (signal processing).

- Small commercial companies seeking the competitive advantage of high reliability software in their market niche.

- Major commercial players who develop and market-leading software packages in industry segments, such as chemical engineering, structural engineering, financial modeling, chip and circuit design, supply chain planning, industrial process engineering, and anything else one can name.

ISL targets the developers of software for use in these areas.

\section{Conclusions}

Initially, ISL intends to be a single source for as large a body of existing interval routines as resources allow. In the longer term, ISL offers a quality, portable, uniform, comprehensive, problem-solving library. Eventually, we aspire to be seamlessly integrated with tools and libraries for approximate computation.

\section{Acknowledgement}

An initial draft of this paper was prepared during a visit of the first author to Tibor Csendes at the Department of Applied Informatics, University of Szeged, Hungary, January 16 - 20, 2006. We thank Tibor for his kind hospitality.

\section{References}

1. Moore, R.E.: Methods and Applications of Interval Analysis. SIAM, Philadelphia, PA (1979) 
2. Neumaier, A.: Interval Methods for Systems of Equations. Cambridge University Press, Cambridge (1990)

3. Fong, K., Jefferson, T., Suyehiro, T., Walton, L.: Guide to the SLATEC common mathematical library. Technical report, netlib.org (1990) See http://www. netlib.org/slatec/.

4. Press, W.H., Flannery, B.P., Teukolsky, S.A., Vetterling, W.T.: Numerical Recipes in Fortran: The Art of Scientific Computing, Second ed. Cambridge University Press, Cambridge (1992) Also available for Fortran 90, C, and C++.

5. Press, W.H., Teukolsky, S.A., Vetterling, W.T., Flannery, B.P.: Numerical Recipes in $\mathrm{C}++$ : The Art of Scientific Computing, Second ed. Cambridge University Press, Cambridge (2002)

6. GSL: GNU Scientific Library (1996 - June 2004) http://www .gnu .org/software/ gsl/.

7. Brönnimann, H., Melquiond, G., Pion, S.: A proposal to add interval arithmetic to the C++ standard library. Technical Report N1843-05-0103 (2005)

8. Knüppel, O.: PROFIL/BIAS web page (2006) http://www.ti3.tu-harburg.de/ Sof tware/PROFILEnglisch.html.

9. Lerch, M., Tischler, G., Wolff von Gudenberg, J., Hofschuster, W., Krämer, W.: The interval library filib ++2.0 - design, features and sample programs. Preprint 2001/4, Universität Wuppertal, Wuppertal, Germany (2001)

10. Melquiond, G., Pion, S., Brönnimann, H.: Boost Interval Arithmetic Library web page (2004) http://www. boost.org/libs/numeric/interval/doc/interval.htm.

11. Goualard, F.: Gaol: NOT Just Another Interval Library web page (2006) http: //sourceforge.net/projects/gaol.

12. Hammer, R., Hocks, M., Kulisch, U., Ratz, D.: Numerical Toolbox for Verified Computing I — Basic Numerical Problems. Springer-Verlag, Heidelberg (1993)

13. Hofschuster, W.: C-XSC - A C++ Class Library web page (2004) http://www . math.uni-wuppertal.de/wrswt/xsc/cxsc.html.

14. Hofschuster, W., Krämer, W.: C-XSC 2.0: A C++ library for extended scientific computing. In: Numerical Software with Result Verification. Number 2991 in Lecture Notes in Computer Science. Springer-Verlag, Heidelberg (2004) 15-35 Also appeared as Preprint BUW-WRSWT 2003/5, Universität Wuppertal, 2003.

15. Hofschuster, W., Krämer, W., Wedner, S., Wiethoff, A.: C-XSC 2.0: A C++ library for extended scientific computing. Preprint BUGHW-WRSWT 2001/1, Universität Wuppertal (2001)

16. Klatte, R., Kulisch, U., Wiethoff, A., Lawo, C., Rauch, M.: C-XSC - A C++ Library for Extended Scientific Computing. Springer-Verlag, Heidelberg (1993)

17. Sun Microsystems: Sun Studio C/C++/Fortran Compilers web page (2006) http: //developers.sun.com/prodtech/cc/compilers_index.html.

18. Anderson, E., Bai, Z., Bischof, C., Blackford, S., Demmel, J., Dongarra, J., Du Croz, J., Greenbaum, A., Hammarling, S., McKenney, A., Sorensen, D.: LAPACK User's Guide, 3rd edn. SIAM, Philadelphia, PA (1999) Certain derivative work portions have been copyrighted by the Numerical Algorithms Group Ltd. See http: //www.netlib.org/lapack/, http://www.nacse.org/demos/lapack/.

19. Balay, S., Buschelman, K., Eijkhout, V., Gropp, W.D., Kaushik, D., Knepley, M.G., McInnes, L.C., Smith, B.F., Zhang, H.: PETSc users manual. Technical Report ANL-95/11 - Revision 2.1.5, Argonne National Laboratory (2004)

20. Balay, S., Buschelman, K., Gropp, W.D., Kaushik, D., Knepley, M.G., McInnes, L.C., Smith, B.F., Zhang, H.: PETSc Web page (2001) http://www.mcs .anl.gov/ petsc. 
21. Balay, S., Gropp, W.D., McInnes, L.C., Smith, B.F.: Efficient management of parallelism in object oriented numerical software libraries. In Arge, E., Bruaset, A.M., Langtangen, H.P., eds.: Modern Software Tools in Scientific Computing, Birkhäuser Press (1997) 163-202

22. Kreinovich, V.: Interval Web page (2006) http://www.cs.utep.edu/ interval-comp/main.html.

23. Parnas, D.L.: Software Fundamentals: Collected Papers by David L. Parnas. Addison-Wesley (2001)

24. Knuth, D.E.: Literate programming. The Computer Journal 27(2) (1984) 97-111

25. LiterateProgramming: Literate Programming Web page (2000-2005) http://www . literateprogramming.com/.

26. Burke, E.M., Coyner, B.M.: Java Extreme Programming Cookbook. O'Reilly, Sebastopol, CA (2003)

27. Beck, K.: Test-Driven Development: By Example. Addison Wesley (2003)

28. Hull, T., Enright, W., Fellen, B., Sedgwick, A.: Comparing numerical methods for ordinary differential equations. SIAM J. Numer. Anal. 9 (1972) 603-637

29. Mazzia, F., Iavernaro, F., Magherini, C.: Test set for IVP solvers, release 2.2 (2003) http://pitagora.dm.uniba.it/ testset/.

30. Pryce, J.D.: A test package for Sturm-Liouville solvers. ACM Trans. Math. Software 25(1) (1999) 21-57

31. Pryce, J.D.: Algorithm 789: SLTSTPAK, a test package for Sturm-Liouville solvers. ACM Trans. Math. Software 25(1) (1999) 58-69

32. Corliss, G.F., Yu, J.: Testing COSY's interval and Taylor model arithmetic. In Alt, R., Frommer, A., Kearfott, R.B., Luther, W., eds.: Numerical Software with Result Verification: Platforms, Algorithms, Applications in Engineering, Physics, and Economics. Number 2992 in Lectures Notes in Computer Science. SpringerVerlag, Heidelburg (2004) 91-105 